ÉDITORIAL

\title{
Où allons-nous avec les traitements de la SEP? Les coûts vont-ils continuer à augmenter ?*
}

Can. J. Neurol. Sci. 2010; 37: 307-308

L'article de Dalia Rotstein, Muhannad Mamdani et Paul O'Connor de la clinique de SEP à l'hôpital St Michael de Toronto publié dans ce numéro du Journal canadien des Sciences neurologiques ${ }^{1}$ est très intéressant sur deux plans : l'utilisation des médicaments immunomodulateurs pour traiter la SEP ne cesse d'augmenter ; l'utilisation de ces médicaments varie grandement d'une province à l'autre.

Il s'agit de l'analyse d'une étude de cohorte examinant l'évolution quantitative et géographique de la prescription d'immunomodulateurs pour la SEP, et l'augmentation du coût total dans les 10 Provinces du Canada entre 2001 et 2007.

A partir des données CompuScript obtenues par la surveillance de pharmacies sur l'ensemble du pays, Rotstein et col. décrivent que la vente des quatre immunomodulateurs utilisés pour la SEP (Beta-1a Interferon IM ou Avonex ${ }^{\circledR}$, Beta1 b Interferon ou Betaseron ${ }^{\circledR}$, Glatiramer Acetate ou Copaxone ${ }^{\circledR}$ et Beta-1a Interferon S.Cu ou Rebif $®$ ) a augmenté de $50 \%$ en 5 ans. Durant cette période, leur coût est passé de 15 à 28 millions de dollars par année. Et ceci malgré leur efficacité limitée, la fréquence des effets secondaires et le fait qu'ils n'existent que sous forme injectable.

Il est certain que l'efficacité des immunomodulateurs est limitée : ils réduisent d'environ $30 \%$ la fréquence des rechutes, ils retardent le début de la phase progressive mais n'ont aucun effet sur la progression du handicap une fois que le patient a atteint la phase secondaire progressive. Ils ont de nombreux effets secondaires qui ne mettent pas en danger les jours du patient mais qui ont des répercussions sur sa qualité de vie : réactions au point d'injection, symptômes pseudo-grippaux après l'injection. Plus rarement ils causent des céphalées tenaces, des problèmes de maturation des cellules sanguines ou l'augmentation des enzymes hépatiques. Un autre inconvénient est leur méthode d'administration, car ils sont tous injectables. En fait ces médicaments sont remarquables par leur absence de complications léthales. ${ }^{2}$ Pourquoi donc continuent-ils à être de plus en plus prescrits d'une façon disproportionnée avec la simple augmentation de la population? Il y a sans doute plusieurs raisons.

Chose étonnante pour des médicaments coûtant si cher, il n'y a pas de règles établies pour determiner quand arrêter le traitement alors que des normes ont été instituées en Europe et en Australie recommandant d'interrompre le traitement en cas de taux élevé d'anticorps neutralisants - dont on sait qu'ils inhibent l'action du medicament- ou en cas d'inefficacité. De plus, des efforts pour définir les bénéfices potentiels des médicaments ont abouti à élargir leurs indications jusqu'à inclure le premier épisode démyélinisant et même la phase secondaire progressive. On peut se demander ce qui va se passer quand de nouveaux médicaments plus pratiques à utiliser seront autorisés sur le marché.

En fait nous avons maintenant deux médicaments plus efficaces que les immunomodulateurs : le Mitoxantone ou Novantrone® (non qualifié pour la SEP) et le Natalizumab (ou Tysabri ${ }^{\circledR}$ ), ils diminuent tous deux le taux de rechutes mieux que les immunomodulateurs; malheureusement ils provoquent aussi des effets secondaires rares mais graves et potentiellement létaux. Une leuco-encéphalopathie progressive multifocale a été observée chez $1 / 2000$ patients traités au Tysabri ${ }^{\circledR}$ et une leucémie chez 1/1000 patients traités au Mitoxantrone. Du simple fait qu'elles ont été reconnues, ces complications vont probablement limiter l'utilisation de ces 2 médicaments pour la SEP. Cependant de nouvelles tendances vont apparaître lorsque des médicaments à prendre oralement vont sortir sur le marché, ce qui va se produire dans un avenir proche.

Le Fingolimod $®$, forme orale à prendre une fois par jour vient de prouver une efficacité supérieure à celle d'Avonex ${ }^{\circledR}{ }^{3}$, et la Cladribine ${ }^{\circledR}{ }^{4}$ forme orale a montré un bénéfice certain comparé au placebo. Tous deux sont des médicaments à prendre oralement, ce qui peut en faciliter la prescription. Une différence importante, c'est que la Cladribine ${ }^{\circledR}$ peut n'être prise qu'une fois par an. Il reste à voir si l'utilisation des immunomodulateurs va diminuer lorsque ces médicaments seront autorisés et mis sur le marché. Ceci dépendra probablement de leur prix. Il faut noter qu'un troisième concurrent est encore en phase III des essais thérapeutiques : le Teriflunomide ${ }^{\circledR}$. Il semble que la manière la moins risquée de limiter l'augmentation du prix des immunomodulateurs va être d'instaurer des règles d'arrêt et d'admettre sur le marché des médicaments à prise orale qui devraient être moins chers (si, et seulement si, ils sont moins chers). Néanmoins la fréquence de leurs effets secondaires à long terme n'est pas encore clairement déterminée.

La deuxième contribution importante de ce rapport pour la compréhencion du contexte canadien est l'utilisation inégale de ces immunomodulateurs d'une province à l'autre. D'après leurs résultats, le nombre de doses mensuelles vendues par an au Nouveau Brunwick $(9,9 / 1000$ habitants) est trois fois plus important qu'en Colombie Britannique (3,3). Il est élevé aussi au Manitoba $(9,0)$, en Saskatchewan $(7,3)$ et au Québec $(7,0)$. C'est en Nouvelle Ecosse qu'il est le plus bas (2).

La Loi canadienne sur la Santé de 1984 et le Cadre visant à améliorer l'Union sociale pour les Canadiens de 1999 ont fixé les 5 principes auxquels le gouvernement du Canada s'est engagé : "intégralité, universalité, transférabilité, gestion publique et accessibilité" 5 . Les résultats de Rotstein et col. sont de toute évidence incompatibles avec 3 de ces principes. La divergence vient probablement du fait que les différentes provinces ont 
établi des directives qui limitent de manière différente la prescription de ces médicaments. La Colombie Brtannique, par exemple, n'autorise pas le remboursement des immunomodulateurs pour le premier évènement démyélinisant, alors que le Québec l'autorise. Il est intéressant de noter que les restrictions concernant la prescription des médicaments pour l'insuffisance cardiaque suivent à peu près le même modèle que celui décrit dans le papier de Rotstein ${ }^{6}$. A noter que ces différences entre provinces n'apparaissent pas dans la prescription des somnifères, ce qui montre le poids de l'argument économique sur la prise de décision des politiques ${ }^{7}$. Quelque part, il est difficile d'admettre que la raison derrière l'indication de traiter ou de ne pas traiter un patient donné avec certains médicaments est déterminée pour au moins $50 \%$ par des facteurs économiques.

Comme il arrive avec beaucoup d'études rétrospectives, cette étude a des limites. Les données sur les changements de prévalence de la SEP au fil des années sont très limitées. Ceci, ajouté au fait que certains patients peuvent avoir eu des prescriptions multiples, a rendu impossible le calcul du pourcentage exact de patients recevant des immunomodulateurs. Cela a aussi rendu moins précis le calcul de l'augmentation du taux de prescription d'un médicament, bien que ceci n'ait probablement pas un impact important, puisque l'augmentation de $59 \%$ est beaucoup plus importante que ce qu'on attend pour le changement de prévalence de la SEP, ont conclu les auteurs.

Cette étude est néanmoins très intéressante pour les neurologues ainsi que pour les prestataires et planificateurs de soins de santé. Elle permet de mieux comprendre les mécanismes qui entrent en jeu dans la manière de prescrire et l'attitude des neurologues délivrant les ordonnances. Cette prise de conscience peut se montrer utile pour concevoir par la suite des indications sur qui traiter, quand traiter, et quel immunomodulateur utiliser. De plus, cette étude confirme que la SEP pèse de plus en plus lourd sur le système canadien des soins de santé, et elle pourrait aider à mieux prévoir l'allocation des fonds. Elle demande aussi que de meilleures assurances médicales soient conçues pour les patients qui ont une SEP.

* Traduit de l'anglais par Marie-Louise Miginiac

Joel Oger, Mona AlKhawajah Vancouver, Colombie Britanique, Canada

\section{RÉFÉRENCES}

1. Rotstein DL, Mamdani M, O'Connor PW. Increasing use of disease modifying drugs for MS in Canada. Can J Neurol Sci. 2010; 37: 383-8.

2. Tremlett HL, Oger J. Ten years of adverse drug reaction reports for the multiple sclerosis immunomodulatory therapies: a Canadian perspective. Mult Scler. 2008 Jan;14(1):94-105.

3. Cohen JA, Barkhof F, Comi G, Hartung HP, Khatri BO, Montalban $\mathrm{X}$, et al. Oral fingolimod or intramuscular interferon for relapsing multiple Sclerosis. N Engl J Med. 2010;362(5):402-15.

4. Giovannoni G, Comi G, Cook S, Rammohan K, Rieckmann P, Sorensen PS, et al. A placebo-controlled trial of Oral Cladribine for relapsing MS. N Engl J Med. 2010:362(5):416-26.

5. Government of Canada, Social Union, News release. A framework to improve the social union for Canadians an agreement between the Government of Canada and the Governments of the Provinces February 4, 1999 [cited 2010 Jan 23] Available from: http://www.socialunion.gc.ca/news/024992.html.

6. Thanassoulis G, Karp I, Humphries K, Tu JV, Eisenberg MJ, Pilote L. Impact of restrictive prescription plans on heart failure medication use. Circ Cardiovasc Qual Outcomes. 2009 Sep;2(5): 484-90.

7. Neutel CI, Patten SB. Sleep medication use in Canadian Seniors. Can J Pharmaco. 2009 Fall;16(3):e443-52. 